\title{
Minimal Slant Submanifolds of the smallest dimension in $S$-manifolds
}

\author{
Alfonso Carriazo, Luis M. Fernández and María Belén Hans-Uber
}

\begin{abstract}
We study slant submanifolds of $S$-manifolds with the smallest dimension, specially minimal submanifolds and establish some relations between them and anti-invariant submanifolds in $S$-manifolds, similar to those ones proved by B.-Y. Chen for slant surfaces and totally real surfaces in Kaehler manifolds.
\end{abstract}

\section{Introduction}

Slant immersions in complex geometry were defined by B.-Y. Chen as a natural generalization of both holomorphic and totally real immersions $[4,6]$. Recently, A. Lotta has introduced the notion of slant immersion of a Riemannian manifold into an almost contact metric manifold [8]. Slant submanifolds of Sasakian manifolds have been studied in [2] and a general view about slant immersions can be found in [3].

On the other hand, for manifolds with an $f$-structure, D.E. Blair has introduced $S$-manifolds as the analogue of the Kaehler structure in the almost complex case and of Sasakian structure in the almost contact case [1].

The purpose of the present paper is to study slant submanifolds of $S$-manifolds with the smallest dimension, specially, minimal slant submanifolds. After recalling, in Section 2, some basic ideas of Riemannian geometry, we review, in Section 3, formulas and definitions for metric $f$-manifolds and their submanifolds, which we shall use later. In Section 4 we prove that the smallest dimension of a slant submanifols in an $S$-manifold is $2+s$, where $s$ is denoting the number of structure vector fields of the ambient $S$-manifold (note that $s=0$ for Kaehler manifolds and $s=1$ for Sasakian manifolds) and we give some characterization theorems for these submanifolds in terms

2000 Mathematics Subject Classification: 53C25, 53C40.

Keywords: $S$-manifold, slant submanifold, minimal submanifold, smallest dimension. 
of the covariant derivatives of the $f$-structure projection operators on the submanifold. Finally, in Section 5 we study minimal slant submanifolds of the smallest dimension. In particular, we establish some relations between minimal slant $(2+s)$-dimensional submanifolds and anti-invariant submanifolds in $S$-manifolds, which correspond, in same sense, to those ones proved by B.-Y. Chen in $[4,6]$.

\section{Preliminaries}

In this section, we will recall some fundamental results and formulas concerning Riemannian submanifolds for later use (see, e.g. [5] as a general reference).

Let $M$ be a Riemannian manifold isometrically immersed in a Riemannian manifold $\widetilde{M}$. Let $g$ denote the metric tensor of $\widetilde{M}$ as well as the induced metric tensor on $M$. Let $\mathcal{X}(\widetilde{M})$ be de Lie algebra of tangent vector fields on $\widetilde{M}, \mathcal{X}(M)$ the Lie algebra of tangent vector fields on $M$ and $T^{\perp} M$ the set of vector fields on $\widetilde{M}$ which are normal to $M$, that is, $\mathcal{X}(\widetilde{M})=\mathcal{X}(M) \oplus T^{\perp} M$.

If $\nabla$ y $\widetilde{\nabla}$ denote the Levi-Civita connections of $M$ and $\widetilde{M}$, respectively, the Gauss-Weingarten formulas are given by

$$
\widetilde{\nabla}_{X} Y=\nabla_{X} Y+\sigma(X, Y), \widetilde{\nabla}_{X} V=-A_{V} X+D_{X} V
$$

for any $X, Y \in \mathcal{X}(M)$ and any $V \in T^{\perp} M$, where $D$ is the normal connection, $\sigma$ is the second fundamental form of the immersion and $A_{V}$ is the Weingarten endomorphism associated with $V$. The endomorphisms $A_{V}$ and $\sigma$ are related by

$$
g\left(A_{V} X, Y\right)=g(\sigma(X, Y), V),
$$

for any $X, Y \in \mathcal{X}(M)$ and any $V \in T^{\perp} M$.

The mean curvature vector $H$ is defined by

$$
H=\frac{1}{m} \operatorname{trace} \sigma=\frac{1}{m} \sum_{i=1}^{m} \sigma\left(e_{i}, e_{i}\right),
$$

where $\operatorname{dim} M=m$ and $\left\{e_{1}, \ldots, e_{m}\right\}$ is a local orthonormal basis of $\mathcal{X}(M)$. $M$ is said to be minimal if $H$ vanishes identically or, equivalently, if

$$
\text { trace } A_{V}=0, \quad \text { for any } V \in T^{\perp} M \text {. }
$$

If $\operatorname{dim}(\widetilde{M})=\widetilde{m}$, a local orthonormal basis of $\mathcal{X}(\widetilde{M})$

$$
\left\{e_{1}, \ldots, e_{m}, e_{m+1}, \ldots, e_{\widetilde{m}}\right\}
$$

can be chosen such that, restricted to $M$, the vector fields $e_{1}, \ldots, e_{m}$ are tangent to $M$ and so, $e_{m+1}, \ldots, e_{\widetilde{m}}$ are normal to $M$. 
Then, for any $X \in \mathcal{X}(M)$, it can be written that

$$
\begin{aligned}
\widetilde{\nabla}_{X} e_{i} & =\sum_{j=1}^{m} w_{i}^{j}(X) e_{j}+\sum_{k=m+1}^{\widetilde{m}} w_{i}^{k}(X) e_{k}, \\
\widetilde{\nabla}_{X} e_{r} & =\sum_{j=1}^{m} w_{r}^{j}(X) e_{j}+\sum_{k=m+1}^{\widetilde{m}} w_{r}^{k}(X) e_{k},
\end{aligned}
$$

for $i \in\{1, \ldots, m\}$ and $r \in\{m+1, \ldots, \widetilde{m}\}$. The 1 -forms $w_{i}^{j}, w_{i}^{k}, w_{r}^{k}$ given by equations (2.1) and (2.2) are called connection forms of $M$ in $\widetilde{M}$. It is easy to show that

$$
w_{j}^{i}+w_{i}^{j}=0, \quad \text { for any } i, j \in\{1, \ldots, m\} .
$$

\section{Slant submanifolds of $S$-manifolds}

Let $(\widetilde{M}, g)$ be a $(2 m+s)$-dimensional Riemannian manifold. Then, it is said to be a metric $f$-manifold if there exist on $\widetilde{M}$ an $f$-structure $f$, that is, a tensor field $f$ of type $(1,1)$ satisfying $f^{3}+f=0$ (see [9]), of rank $2 m$ and $s$ global vector fields $\xi_{1}, \ldots, \xi_{s}$ (called structure vector fields) such that, if $\eta_{1}, \ldots, \eta_{s}$ are the dual 1 -forms of $\xi_{1}, \ldots, \xi_{s}$, then

$$
\begin{aligned}
& f \xi_{\alpha}=0 ; \quad \eta_{\alpha} \circ f=0 ; \quad f^{2}=-I+\sum_{\alpha=1}^{s} \eta_{\alpha} \otimes \xi_{\alpha} \\
& g(X, Y)=g(f X, f Y)+\sum_{\alpha=1}^{s} \eta_{\alpha}(X) \eta_{\alpha}(Y)
\end{aligned}
$$

for any $X, Y \in \mathcal{X}(\widetilde{M})$ and $\alpha=1, \ldots, s$.

The $f$-structure $f$ is normal if

$$
[f, f]+2 \sum_{\alpha=1}^{s} \xi_{\alpha} \otimes \mathrm{d} \eta_{\alpha}=0
$$

where $[f, f]$ is the Nijenhuis tensor of $f$. Let $F$ be the fundamental 2-form defined by $F(X, Y)=g(X, f Y)$, for any $X, Y \in \mathcal{X}(\widetilde{M})$. Then, $\widetilde{M}$ is said to be an $S$-manifold if the $f$-structure is normal and

$$
\eta_{1} \wedge \cdots \wedge \eta_{s} \wedge\left(\mathrm{d} \eta_{\alpha}\right)^{n} \neq 0, F=\mathrm{d} \eta_{a}
$$

for any $\alpha=1, \ldots, s$. In this case, the structure vector fields are Killing vector fields. When $s=1, S$-manifolds are Sasakian manifolds. 
The Riemannian connection $\widetilde{\nabla}$ of an $S$-manifold satisfies ([1])

$$
\widetilde{\nabla}_{X} \xi_{\alpha}=-f X
$$

and

$$
\left(\widetilde{\nabla}_{X} f\right) Y=\sum_{\alpha=1}^{s}\left(g(f X, f Y) \xi_{\alpha}+\eta_{\alpha}(Y) f^{2} X\right),
$$

for any $X, Y \in \mathcal{X}(\widetilde{M})$ and any $\alpha=1, \ldots, s$.

Next, let $M$ be a isometrically immersed submanifold of a metric $f$-manifold $\widetilde{M}$. For any $X \in \mathcal{X}(M)$ we write

$$
f X=T X+N X
$$

where $T X$ and $N X$ are the tangential and normal components of $f X$, respectively. Similarly, for any $V \in T^{\perp} M$, we have

$$
f V=t V+n V
$$

where $t V$ (resp., $n V$ ) is the tangential component (resp., the normal component) of $f V$. Since, from (3.1), the metric $g$ satisfies that $g(f X, Y)=$ $-g(X, f Y)$, for any $X, Y \in \mathcal{X}(\widetilde{M})$, by using (3.4) and (3.5), we get

$$
\begin{aligned}
g(T X, Y) & =-g(X, T Y), \\
g(n V, U) & =-g(V, n U), \\
g(N X, V) & =-g(X, T V),
\end{aligned}
$$

for any $X, Y \in \mathcal{X}(M), U, V \in T^{\perp} M$ and, by using (3.5), if the structure vector fields are tangent to $M$,

$$
N T X+n N X=0
$$

for any $X \in \mathcal{X}(M)$. Moreover, in this last case, if $\widetilde{M}$ is an $S$-manifold, from (3.2) and (3.4) it is easy to show that

$$
\sigma\left(X, \xi_{\alpha}\right)=-N X
$$

for any $X \in \mathcal{X}(M), \alpha=1, \ldots, s$ and, consequently $\sigma\left(\xi_{\alpha}, \xi_{\beta}\right)=0$, for any $\alpha, \beta=1, \ldots, s$.

The covariant derivatives of $T$ and $N$ are given by

$$
\begin{aligned}
\left(\nabla_{X} T\right) Y & =\nabla_{X} T Y-T \nabla_{X} Y \\
\left(\nabla_{X} N\right) Y & =D_{X} N Y-N \nabla_{X} Y
\end{aligned}
$$

for any $X, Y \in \mathcal{X}(M)$. 
Then, by using (3.3), (3.11), (3.12) and Gauss-Weingarten formulas, it can be obtained that

$$
\begin{aligned}
& \left(\nabla_{X} T\right) Y=t \sigma(X, Y)+A_{N Y} X+\sum_{\alpha=1}^{s}\left(g(f X, f Y) \xi_{\alpha}+\eta_{\alpha}(Y) f^{2} X\right), \\
& \left(\nabla_{X} N\right) Y=n \sigma(X, Y)-\sigma(X, T Y)
\end{aligned}
$$

for any $X, Y \in \mathcal{X}(M)$.

Now, for later use, we establish two general lemmas for submanifolds of $S$-manifolds which can be proved from (2.1) and (3.6)-(3.8) by a straightforward computation:

Lemma 3.1 Let $M$ be a submanifold of an $S$-manifold, tangent to the structure vector fields. Then, there exists a differentiable function $\lambda$ such that

$$
\left(\nabla_{X} T\right) Y=\lambda \sum_{\alpha=1}^{s}\left(g(f X, f Y) \xi_{\alpha}+\eta_{\alpha}(Y) f^{2} X\right)
$$

for any $X, Y \in \mathcal{X}(M)$, if and only if:

$$
A_{N Y} X-A_{N X} Y=(\lambda-1) \sum_{\alpha=1}^{s}\left(\eta_{\alpha}(Y) f^{2} X-\eta_{\alpha}(X) f^{2} Y\right) \text {. }
$$

Lemma 3.2 Let $M$ be a submanifold of an $S$-manifold, tangent to the structure vector fields. Then,

$$
\left(\nabla_{X} N\right) Y=\sum_{\alpha=1}^{s}\left(2 \eta_{\alpha}(X) N T Y+\eta_{\alpha}(Y) N T X\right),
$$

for any $X, Y \in \mathcal{X}(M)$, if and only if:

$$
A_{V} T Y+A_{n V} Y=\sum_{\alpha=1}^{s}\left(2 g(Y, \operatorname{tn} V) \xi_{\alpha}+\eta_{\alpha}(Y) \operatorname{tn} V\right),
$$

for any $Y \in \mathcal{X}(M)$ and any $V \in T^{\perp} M$.

The submanifold $M$ is said to be invariant if $N$ is identically zero, that is, if $f X \in \mathcal{X}(M)$, for any $X \in \mathcal{X}(M)$. On the other hand, $M$ is said to be an anti-invariant submanifold if $T$ is identically zero, that is, if $f X \in T^{\perp} M$, for any $X \in \mathcal{X}(M)$.

From now on, we suppose that all the structure vector fields are tangent to the submanifold $M$. Then, $M$ is said to be a slant submanifold if for any $x \in M$ and any $X \in T_{x} M$, linearly independent on $\xi_{1}, \ldots, \xi_{s}$, the Wirtinger angle between $f X$ and $T_{x} M$ is a constant $\theta \in[0, \pi / 2]$, called the slant angle of $M$ in $\widetilde{M}$. Note that this definition generalizes that one given by B.-Y. Chen ([6]) for Complex Geometry and that one given by A. Lotta ([8]) for Contact Geometry. 
Furthermore, invariant and anti-invariant submanifolds are slant submanifolds with slant angle $\theta=0$ and $\theta=\pi / 2$, respectively. A slant immersion which is not invariant nor anti-invariant is called a proper slant immersion. Observe that, for invariant submanifolds, $T=f$ and, so

$$
T^{2}=f^{2}=-I+\sum_{\alpha=1}^{s} \eta_{\alpha} \otimes \xi_{\alpha},
$$

while for anti-invariant submanifolds, $T^{2}=0$. In fact, we have the following general result whose proof can be obtained by following the same steps as in the case $s=1$ (see [2]):

Theorem 3.1 Let $M$ be a submanifold of a metric $f$-manifold $\widetilde{M}$, tangent to the structure vector fields. Then, $M$ is a slant submanifold if and only if there exists a constant $\lambda \in[0,1]$ such that:

$$
T^{2}=-\lambda I+\lambda \sum_{\alpha=1}^{s} \eta_{\alpha} \otimes \xi_{\alpha}=\lambda f^{2} .
$$

Furthermore, in such case, if $\theta$ is the slant angle of $M$, it satisfies that $\lambda=\cos ^{2} \theta$.

Using (3.1), (3.4), (3.6) and Theorem 3.1, a direct computation gives:

Corollary 3.1 Let $M$ be a slant submanifold of a metric $f$-manifold $\widetilde{M}$, with slant angle $\theta$. Then, for any $X, Y \in \mathcal{X}(M)$, we have:

$$
\begin{aligned}
& g(T X, T Y)=\cos ^{2} \theta\left(g(X, Y)-\sum_{\alpha=1}^{s} \eta_{\alpha}(X) \eta_{\alpha}(Y)\right), \\
& g(N X, N Y)=\sin ^{2} \theta\left(g(X, Y)-\sum_{\alpha=1}^{s} \eta_{\alpha}(X) \eta_{\alpha}(Y)\right) .
\end{aligned}
$$

We also have:

Corollary 3.2 Let $M$ be a non-invariant slant $(m+s)$-dimensional submanifold of a $(2 m+s)$-dimensional metric $f$-manifold $\widetilde{M}$ with slant angle $\theta$ and let $\left\{e_{1}, \ldots, e_{m}, \xi_{1}, \ldots, \xi_{s}\right\}$ be a local orthonormal basis of $\mathcal{X}(M)$. Then,

$$
\left\{(\csc \theta) N e_{1}, \ldots,(\csc \theta) N e_{m}\right\}
$$

is a local orthonormal basis of $T^{\perp} M$.

Proof. It is easy to show that $\left\{(\csc \theta) N e_{1}, \ldots,(\csc \theta) N e_{m}\right\}$ is a set of $m$ linearly independent vector fields of $T^{\perp} M$, that is, a local basis of $T^{\perp} M$. Moreover, from Corollary 3.1, we obtain that:

$$
g\left((\csc \theta) N e_{i},(\csc \theta) N e_{j}\right)=\csc ^{2} \theta \sin ^{2} \theta g\left(e_{i}, e_{j}\right)=\delta_{i j} .
$$


In a similar way, by using Theorem 3.1 and Corollary 3.1, we get:

Corollary 3.3 Let $M$ be a non anti-invariant $(2+s)$-dimensional slant submanifold of a metric $f$-manifold with slant angle $\theta$. Let $e_{1}$ be a unit vector field, tangent to $M$ and normal to the structure vector fields and define $e_{2}=(\sec \theta) T e_{1}$. Then $e_{1}=-(\sec \theta) T e_{2}$ and $\left\{e_{1}, e_{2}, \xi_{1}, \ldots, \xi_{s}\right\}$ is a local orthonormal basis of $\mathcal{X}(M)$.

Finally, combining Corollary 3.2 and Corollary 3.3 and using Theorem 3.1 again, we obtain:

Corollary 3.4 Let $M$ be a proper $(2+s)$-dimensional slant submanifold of a $(4+s)$-dimensional metric $f$-manifold with slant angle $\theta$. Let $e_{1}$ be a unit vector field, tangent to $M$ and normal to the structure vector fields and define:

$$
e_{2}=(\sec \theta) T e_{1}, e_{3}=(\csc \theta) N e_{1} \text { and } e_{4}=(\csc \theta) N e_{2} .
$$

Then, $e_{1}=-(\sec \theta) T e_{2}$ and $\left\{e_{1}, e_{2}, e_{3}, e_{4}, \xi_{1}, \ldots, \xi_{s}\right\}$ is a local orthonormal basis of $\mathcal{X}(\widetilde{M})$ such that $e_{1}, e_{2}, \xi_{1}, \ldots, \xi_{s}$ are tangent to $M$ and $e_{3}, e_{4}$ are normal to $M$. Moreover:

$$
t e_{3}=-\sin \theta e_{1}, n e_{3}=-\cos \theta e_{4}, t e_{4}=-\sin \theta e_{2}, n e_{4}=\cos \theta e_{3} .
$$

The basis $\left\{e_{1}, e_{2}, e_{3}, e_{4}, \xi_{1}, \ldots, \xi_{s}\right\}$ is said to be an adapted slant basis.

\section{Slant submanifolds of the smallest dimension}

Observe that $2+s$ is the smallest dimension of a proper slant submanifold in a metric $f$-manifold. Indeed, if we denote $Q=T^{2}$ and consider the orthogonal decomposition

$$
\mathcal{X}(M)=\mathcal{L} \oplus \mathcal{M}
$$

where $\mathcal{M}$ is the distribution spanned by the structure vector fields and $\mathcal{L}$ is its complementary orthogonal distribution, then, since $T \mathcal{L} \subseteq \mathcal{L},\left.Q\right|_{\mathcal{L}}$ is an endomorphism on $\mathcal{L}$. Furthermore, it is a symmetric endomorphism because, from (3.6),

$$
g(Q X, Y)=g\left(T^{2} X, Y\right)=-g(T X, T Y)=g\left(X, T^{2} Y\right)=g(X, Q Y),
$$

for any $X, Y \in \mathcal{X}(M)$. Consequently, for each $x \in M$, the subspace $\mathcal{L}_{x}$ of $T_{x} M$ admits a decomposition of the form

$$
\mathcal{L}_{x}=\mathcal{L}_{x}^{1} \oplus \mathcal{L}_{x}^{2} \oplus \cdots \oplus \mathcal{L}_{x}^{k(x)},
$$

where $\mathcal{L}_{x}^{i}$ is the proper subspace of eigenvectors associated with an eigenvalue $\lambda_{i}$ of $\left.Q\right|_{\mathcal{L}}$. Then, we can easily prove: 
Proposition 4.1 Let $M$ be a submanifold of a metric $f$-manifold, tangent to the structure vector fields. Then, at each point of $M$, we have the following properties:

1. $\lambda_{i} \in[-1,0]$, for any eigenvalue $\lambda_{i}$ of $\left.Q\right|_{\mathcal{L}}$.

2. $T X \in \mathcal{L}^{i}$, for any $X \in \mathcal{L}^{i}$.

3. If $\lambda_{i} \neq 0, \mathcal{L}^{i}$ is of even dimension and $T\left(\mathcal{L}^{i}\right)=\mathcal{L}^{i}$.

Corollary 4.1 Let $M$ be a $(1+s)$-dimensional submanifold of a metric $f$ manifold, tangent to the structure vector fields. Then, $M$ is an anti-invariant submanifold.

Proof. Since $\mathcal{L}$ is of odd dimension (equal to 1 ), from Proposition 4.1 we get $\lambda=0$ and $M$ is an anti-invariant submanifold.

From this corollary, we deduce that there are not proper slant submanifolds of a metric $f$-manifold of dimension smaller than $2+s$. Now, we are going to study submanifolds of such dimension when the ambient manifold is an $S$-manifold. First, by using Theorem 3.1, if $M$ is a slant submanifold with slant angle $\theta$, a direct calculation gives

$$
\left(\nabla_{X} Q\right) Y=\cos ^{2} \theta \sum_{\alpha=1}^{s}\left(g(X, T Y) \xi_{\alpha}-\eta_{\alpha}(Y) T X\right)
$$

for any $X, Y \in \mathcal{X}(M)$, where we recall that

$$
\left(\nabla_{X} Q\right) Y=\nabla_{X} Q Y-Q \nabla_{X} Y .
$$

Next, we have the following general characterization:

Theorem 4.1 Let $M$ be a submanifold of an S-manifold, tangent to the structure vector fields. Then, $M$ is a slant submanifold if and only if the following conditions are satisfied:

1. The endomorphism $\left.Q\right|_{\mathcal{L}}$ has only one eigenvalue at any point of $M$.

2. There exists a function $\lambda: M \longrightarrow[0,1]$ such that

$$
\left(\nabla_{X} Q\right) Y=\lambda \sum_{\alpha=1}^{s}\left(g(X, T Y) \xi_{\alpha}-\eta_{\alpha}(Y) T X\right),
$$

for any $X, Y \in \mathcal{X}(M)$.

Moreover, in this case, if $\theta$ is the slant angle of $M$, then $\lambda=\cos ^{2} \theta$. 
Proof. If $M$ is a slant submanifold with slant angle $\theta$, from Theorem 3.1, we have

$$
T^{2} X=Q X=\cos ^{2} \theta f^{2} X
$$

for any $X \in \mathcal{X}(M)$. Then, $\left.Q\right|_{\mathcal{L}}=-\cos ^{2} \theta I$ and $\lambda_{1}=-\cos ^{2} \theta$ is the only eigenvalue of $\left.Q\right|_{\mathcal{L}}$ at any point of $M$. Furthermore, Condition 2 is (4.1).

Conversely, let $\lambda_{1}(x)$ be the only eigenvalue of $\left.Q\right|_{\mathcal{L}}$ at any point $x \in M$. Thus, by using Condition 2 we get that $\lambda_{1}$ is a constant. Now, let $X \in \mathcal{X}(M)$. If we put

$$
X=\widetilde{X}+\sum_{\alpha=1}^{s} \eta_{\alpha}(X) \xi_{a}
$$

where $\widetilde{X} \in \mathcal{L}$, then $Q X=Q \widetilde{X}=\lambda_{1} \widetilde{X}$ and, so:

$$
Q X=\lambda_{1} X-\lambda_{1} \sum_{\alpha=1}^{s} \eta_{\alpha}(X) \xi_{a} .
$$

By applying Theorem 3.1 we obtain that $M$ is a slant submanifold and, by (4.1), $\lambda=-\lambda_{1}=\cos ^{2} \theta$.

Corollary 4.2 Let $M$ be a $(2+s)$-dimensional submanifold of an $S$-manifold tangent to the structure vector fields. Then, $M$ is a slant submanifold if and only if there exists a function $\lambda: M \longrightarrow[0,1]$ such that

$$
\left(\nabla_{X} Q\right) Y=\lambda \sum_{\alpha=1}^{s}\left(g(X, T Y) \xi_{\alpha}-\eta_{\alpha}(Y) T X\right),
$$

for any $X, Y \in \mathcal{X}(M)$. Moreover, in this case, if $\theta$ is the slant angle of $M$, then $\lambda=\cos ^{2} \theta$.

Proof. We only have to prove that $\left.Q\right|_{\mathcal{L}}$ has only one eigenvalue at any point of $M$. But it is a direct consequence of 3. of Proposition 4.1.

Theorem 4.2 Let $M$ be a $(2+s)$-dimensional submanifold of an $S$-manifold, tangent to the structure vector fields. Then, $M$ is a slant submanifold if and only if there exists a function $\lambda: M \longrightarrow[0,1]$ such that

$$
\left(\nabla_{X} T\right) Y=\lambda \sum_{\alpha=1}^{s}\left(g(f X, f Y) \xi_{\alpha}+\eta_{\alpha}(Y) f^{2} X\right)
$$

for any $X, Y \in \mathcal{X}(M)$. Moreover, in this case, if $\theta$ is the slant angle of $M$, then $\lambda=\cos ^{2} \theta$. 
Proof. First, it is easy to show that (4.3) implies (4.2). Then, we only have to apply Corollary 4.2 to get that $M$ is a slant submanifold. Conversely, we can suppose that $M$ is a proper slant submanifold because if $M$ is an invariant or an anti-invariant submanifold, we obtain (4.3) directly. Now, since $\operatorname{dim}(M)=2+s$, from Corollary 3.3, we can choose a local orthonormal basis of $\mathcal{X}(M),\left\{e_{1}, e_{2}, \xi_{1}, \ldots, \xi_{s}\right\}$, such that $e_{2}=(\sec \theta) T e_{1}$ and $e_{1}=-(\sec \theta) T e_{2}$. Thus, for any $X \in \mathcal{X}(M)$, we have

$$
\left(\nabla_{X} T\right) e_{1}=\cos \theta \sum_{\alpha=1}^{s} w_{2}^{\alpha}(X) \xi_{\alpha}
$$

because $w_{i}^{i}(X)=0$ and $w_{i}^{j}(X)=-w_{j}^{i}(X)$. But, by using (3.2) and (3.4), $w_{2}^{\alpha}(X)=g\left(e_{2}, T X\right)$, for any $\alpha=1, \ldots, s$ and so:

$$
\left(\nabla_{X} T\right) e_{1}=\cos \theta \sum_{\alpha=1}^{s} g\left(e_{2}, T X\right) \xi_{\alpha}=\cos ^{2} \theta \sum_{\alpha=1}^{s} g\left(X, e_{1}\right) \xi_{\alpha} .
$$

Similarly:

$$
\left(\nabla_{X} T\right) e_{2}=\cos ^{2} \theta \sum_{\alpha=1}^{s} g\left(X, e_{2}\right) \xi_{\alpha}
$$

On the other hand, for any $\alpha=1, \ldots, s$ :

$$
\left(\nabla_{X} T\right) \xi_{\alpha}=\cos ^{2} \theta f^{2} X
$$

Now, given any $Y \in \mathcal{X}(M)$, since locally

$$
Y=Y_{1} e_{1}+Y_{2} e_{2}+\sum_{\alpha=1}^{s} \eta_{\alpha}(Y) \xi_{\alpha}
$$

we obtain that:

$$
\left(\nabla_{X} T\right) Y=Y_{1}\left(\nabla_{X} T\right) e_{1}+Y_{2}\left(\nabla_{X} T\right) e_{2}+\sum_{\alpha=1}^{s} \eta_{\alpha}(Y)\left(\nabla_{X} T\right) \xi_{\alpha}
$$

Substituting (4.4)-(4.6) into (4.7) we conclude the proof.

From Lemma 3.1 we get:

Corollary 4.3 Let $M$ be a submanifold of dimension $2+s$ in an $S$-manifold, tangent to the structure vector fields. Then, $M$ is a slant submanifold if and only if there exists a differentiable function $\mu: M \longrightarrow[0,1]$ such that

$$
A_{N Y} X-A_{N X} Y=\mu \sum_{\alpha=1}^{s}\left(\eta_{\alpha}(X) f^{2} Y-\eta_{\alpha}(Y) f^{2} X\right),
$$

for any $X, Y \in \mathcal{X}(M)$. Moreover, in this case, if $\theta$ is the slant angle of $M$, then $\mu=\sin ^{2} \theta$. 


\section{Minimal slant submanifolds of the smallest dimension}

For later use, we are going to prove the following lemmas:

Lemma 5.1 Let $M$ be a proper slant, $(2+s)$-dimensional submanifold of an $S$-manifold $\widetilde{M}$ with $\operatorname{dim}(\widetilde{M})=4+s$. If $\theta$ is the slant angle,

$$
\left\{e_{1}, \ldots, e_{4}, e_{5}=\xi_{1}, \ldots, e_{4+s}=\xi_{s}\right\}
$$

is an adapted slant basis and if we put

$$
\sigma_{i j}^{r}=g\left(\sigma\left(e_{i}, e_{j}\right), e_{r}\right), \quad \text { for any } i, j=1,2,5, \ldots, 4+s \text { and } r=3,4,
$$

then:

$$
\begin{gathered}
\sigma_{12}^{3}=\sigma_{11}^{4}, \sigma_{22}^{3}=\sigma_{12}^{4} \\
\sigma_{1(4+\alpha)}^{3}=\sigma_{2(4+\alpha)}^{4}=-\sin \theta, \alpha=1, \ldots, s \\
\sigma_{2(4+\alpha)}^{3}=\sigma_{1(4+\alpha)}^{4}=\sigma_{(4+\alpha)(4+\beta)}^{3}=\sigma_{(4+\alpha)(4+\beta)}^{4}=0, \alpha, \beta=1, \ldots, s .
\end{gathered}
$$

Proof. We obtain (5.1) by virtue of Corollary 4.3 while (5.2) and (5.3) hold because $\widetilde{M}$ is an $S$-manifold.

Lemma 5.2 Let $M$ be a $(2+s)$-dimensional slant submanifold of an $S$ manifold $\widetilde{M}$ with $\operatorname{dim}(\widetilde{M})=4+s$. Then, $\nabla N=0$ if and only if $M$ is either an invariant or an anti-invariant submanifold.

Proof. If $\nabla N=0$, then, by applying (3.14) we get, for any $X, Y \in \mathcal{X}(M)$, $V \in T^{\perp} M$ :

$$
-g(\sigma(X, T Y), V)=g(\sigma(X, Y), n V) .
$$

If we suppose that $M$ is a proper slant submanifold with slant angle $\theta$ and choose an adapted slant basis

$$
\left\{e_{1}, \ldots, e_{4}, e_{5}=\xi_{1}, \ldots, e_{4+s}=\xi_{s}\right\},
$$

then, from (5.4), since $T e_{4+\alpha}=T \xi_{\alpha}=0$, for any $\alpha=1, \ldots, s$ and $n e_{4}=$ $\cos \theta e_{3}$,

$$
\begin{aligned}
0 & =g\left(\sigma\left(e_{1}, e_{4+\alpha}\right), n e_{4}\right)=\cos \theta g\left(\sigma\left(e_{1}, e_{4+\alpha}\right), e_{3}\right)= \\
& =\cos \theta \sigma_{1(4+\alpha)}^{3}=-\cos \theta \sin \theta,
\end{aligned}
$$

where we have used (5.2). But this contradicts the fact of $M$ being a proper slant submanifold.

Conversely, if $M$ is an invariant submanifold, then $N=0$ and so, $\nabla N=0$. Finally, if $M$ is anti-invariant submanifold, then $n=0$ and we only need to apply (3.14). 
Theorem 5.1 Let $M$ be $a(2+s)$-dimensional submanifold of a $(4+s)$ dimensional $S$-manifold $\widetilde{M}$, tangent to the structure vector fields.

1. If $M$ is a minimal proper slant submanifold of $\widetilde{M}$, then

$$
\left(\nabla_{X} N\right) Y=\sum_{\alpha=1}^{s}\left(2 \eta_{\alpha}(X) N T Y+\eta_{\alpha}(Y) N T X\right) .
$$

for any $X, Y \in \mathcal{X}(M)$.

2. Conversely, suppose that there is an eigenvalue $\lambda$ of $\left.Q\right|_{\mathcal{L}}$ at each point of $M$ such that $\lambda \in(-1,0)$. In this case, if (5.5) holds, $M$ is a minimal proper slant submanifold of $\widetilde{M}$.

Proof. To prove statement 1 , we choose an adapted slant basis:

$$
\left\{e_{1}, \ldots, e_{4}, e_{5}=\xi_{1}, \ldots, e_{4+s}=\xi_{s}\right\} \text {. }
$$

Then, we can show that

$$
n \sigma\left(e_{i}, e_{j}\right)=\cos \theta \sigma_{i j}^{4} e_{3}-\cos \theta \sigma_{i j}^{3} e_{4},
$$

for any $i, j=1,2,5, \ldots, 4+s$. Moreover, since $M$ is minimal, by using $\sigma\left(\xi_{a}, \xi_{\alpha}\right)=0$ for any $\alpha=1, \ldots, s$, we have:

$$
\sigma_{11}^{3}=-\sigma_{22}^{3}, \sigma_{11}^{4}=-\sigma_{22}^{4}
$$

Next, writing $X, Y \in \mathcal{X}(M)$ in terms of the adapted slant basis and taking into account (5.1)-(5.3), (5.6) and (5.7), we obtain (5.5) from (3.14) and (3.9).

To prove statement 2 , we can choose a unit local vector field $e_{1}$ in $\mathcal{L}$, such that

$$
T^{2} e_{1}=-\cos ^{2} \theta_{1} e_{1},
$$

where $\theta_{1}=\theta\left(e_{1}\right) \in(0, \pi / 2)$ denotes the Wirtinger angle of $e_{1}$. Now, we define $e_{2}, e_{3}, e_{4}$ by

$$
e_{2}\left(\sec \theta_{1}\right) T e_{1}, e_{3}=\left(\csc \theta_{1}\right) N e_{1}, e_{4}=\left(\csc \theta_{1}\right) N e_{2}
$$

and $e_{4+\alpha}=\xi_{\alpha}, \alpha=1, \ldots, s$. It is easy to show that $\left\{e_{1}, \ldots, e_{4+s}\right\}$ is a local orthonormal basis of $\widetilde{M}$ such that:

$$
t e_{3}=-\sin \theta_{1} e_{1}, t e_{4}=-\sin \theta_{1} e_{2}, n e_{3}=-\cos \theta_{1} e_{4}, n e_{4}=\cos \theta_{1} e_{3} .
$$


Next, from (5.8) and by using Lemma 3.2, we get:

$$
A_{N e_{1}} e_{2}=\sec \theta_{1} \sin \theta_{1} A_{e_{3}} T e_{1}=\sin \theta_{1} A_{e_{4}} e_{1}=A_{N e_{2}} e_{1} .
$$

Furthermore, from (3.2) and Gauss-Weingarten formulas, we have, for any $\alpha=1, \ldots, s$,

$$
A_{N e_{1}} e_{4+\alpha}=\sin \theta_{1} A_{e_{3}} e_{4+\alpha}=\sin \theta_{1} t e_{3}=-\sin ^{2} \theta_{1} e_{1}
$$

and

$$
A_{N e_{2}} e_{4+\alpha}=\sin \theta_{1} A_{e_{4}} e_{4+\alpha}=\sin \theta_{1} t e_{4}=-\sin ^{2} \theta_{1} e_{2} .
$$

Hence, a direct computation gives that

$$
A_{N Y} X=A_{N X} Y-\sin ^{2} \theta_{1} \sum_{\alpha=1}^{s}\left(\eta_{a}(Y) f^{2} X-\eta_{a}(X) f^{2} Y\right),
$$

for any $X, Y \in \mathcal{X}(M)$ and so, by applying Corollary 4.3, we know that $M$ is a proper slant submanifold, with slant angle $\theta_{1}$. Finally, to prove that $M$ is also a minimal submanifold, we only need to show that:

$$
\sigma_{11}^{3}=-\sigma_{22}^{3}, \sigma_{11}^{4}=-\sigma_{22}^{4}
$$

But,

$$
\sigma_{11}^{3}=g\left(\sigma\left(e_{1}, e_{1}\right), e_{3}\right)=\left(-\sec \theta_{1}\right) g\left(\sigma\left(e_{1}, T e_{2}\right), e_{3}\right)
$$

and, from (3.14) y (5.5), $\sigma\left(e_{1}, T e_{2}\right)=n \sigma\left(e_{1}, e_{2}\right)$, which together (3.7) implies:

$$
\sigma_{11}^{3}=-\sigma_{12}^{4} .
$$

Now, since we have already proved that $M$ is a proper slant submanifold and the chosen basis is an adapted slant one, from Lemma 5.1 we conclude the proof.

Note that (5.5) holds directly in the invariant and anti-invariant cases, since $\nabla N=0$. On the other hand, the above theorem is the corresponding one to Theorem 5.5 in [6], proved by B.-Y. Chen for surfaces in 4-dimensional Kaehler manifols.

Next, we want to establish some relations between minimal slant $(2+s)$ dimensional submanifolds and anti-invariant submanifolds in $S$-manifolds. First, we have the following lemma:

Lemma 5.3 Let $M$ be a proper slant $(2+s)$-dimensional submanifold in a $(4+s)$-dimensional $S$-manifold $\widetilde{M}$, with slant angle $\theta$. Then, with respect to an adapted slant basis $\left\{e_{1}, \ldots, e_{4+s}\right\}$, we have

$$
w_{3}^{4}-w_{1}^{2}=-\cot \theta\left(\left(\operatorname{trace} \sigma^{3}\right) w^{1}+\left(\operatorname{trace} \sigma^{4}\right) w^{2}-\sum_{\alpha=1}^{s}(2 \sin \theta) \eta_{\alpha}\right),
$$

where $w^{1}, w^{2}$ are the dual forms of $e_{1}, e_{2}$. 
Proof. Since the local basis is an adapted slant one, then, by using (3.14):

$$
D_{e_{1}} e_{3}=(\csc \theta) D_{e_{1}} N e_{1}=(\csc \theta)\left(N\left(\nabla_{e_{1}} e_{1}\right)+n \sigma\left(e_{1}, e_{1}\right)-\sigma\left(e_{1}, T e_{1}\right)\right) .
$$

But, from (2.2), (2.4) and applying $N$, we get:

$$
N\left(\nabla_{e_{1}} e_{1}\right)=w_{1}^{2}\left(e_{1}\right) N e_{2}=\sin \theta w_{1}^{2}\left(e_{1}\right) e_{4} .
$$

On the other hand:

$$
\begin{aligned}
& n \sigma\left(e_{1}, e_{1}\right)=\sigma_{11}^{3} n e_{3}+\sigma_{11}^{4} n e_{4}=\cos \theta\left(-\sigma_{11}^{3} e_{4}+\sigma_{11}^{4} e_{3}\right), \\
& \sigma\left(e_{1}, T e_{1}\right)=\cos \theta \sigma\left(e_{1}, e_{2}\right)=\cos \theta\left(\sigma_{12}^{3} e_{3}+\sigma_{12}^{4} e_{4}\right) .
\end{aligned}
$$

Substituting (5.11)-(5.13) into (5.10),

$$
D_{e_{1}} e_{3}=w_{1}^{2}\left(e_{1}\right) e_{4}+\cot \theta\left(-\sigma_{11}^{3} e_{4}+\sigma_{11}^{4} e_{3}-\sigma_{12}^{3} e_{3}-\sigma_{12}^{4} e_{4}\right),
$$

by virtue of Lemma 5.1, since

$$
\text { trace } \sigma^{3}=\sum_{i=1}^{2} g\left(\sigma\left(e_{i}, e_{i}\right), e_{3}\right)
$$

we have

$$
D_{e_{1}} e_{3}=w_{1}^{2}\left(e_{1}\right) e_{4}-\cot \theta\left(\operatorname{trace} \sigma^{3}\right) e_{4}
$$

and, from (2.3):

$$
w_{3}^{4}\left(e_{1}\right)-w_{1}^{2}\left(e_{1}\right)=-\cot \theta\left(\operatorname{trace} \sigma^{3}\right) .
$$

Similarly:

$$
w_{3}^{4}\left(e_{2}\right)-w_{1}^{2}\left(e_{2}\right)=-\cot \theta(\operatorname{trace} \sigma) .
$$

Moreover, for any $\alpha=1, \ldots, s$,

$$
D_{e_{4+\alpha}} e_{3}=\csc \theta\left(N\left(\nabla_{\xi_{\alpha}} e_{1}\right)+n \sigma\left(e_{1}, \xi_{\alpha}\right)-\sigma\left(T e_{1}, \xi_{\alpha}\right)\right),
$$

but, by applying (3.9) and (3.10),

$$
n \sigma\left(e_{1}, \xi_{\alpha}\right)-\sigma\left(T e_{1}, \xi_{\alpha}\right)=-n N e_{1}+N T e_{1}=2 N T e_{1},
$$

and, consequently, from Corollary 3.4, we obtain:

$$
n \sigma\left(e_{1}, \xi_{\alpha}\right)-\sigma\left(T e_{1}, \xi_{\alpha}\right)=2 \sin \theta \cos \theta e_{4} .
$$

Furthermore:

$$
N\left(\nabla_{e_{4+\alpha}} e_{1}\right)=w_{1}^{2}\left(e_{4+\alpha}\right) N e_{2}=\sin \theta w_{1}^{2}\left(\xi_{\alpha}\right) e_{4} .
$$


Thus, substituting (5.17) and (5.18) into (5.16) and taking into account that

$$
D_{e_{4+\alpha}} e_{3}=w_{3}^{4}\left(e_{4+\alpha}\right) e_{4},
$$

we get:

$$
w_{3}^{4}\left(e_{4+\alpha}\right)-w_{1}^{2}\left(e_{4+\alpha}\right)=2 \cos \theta=-\cot \theta(-2 \sin \theta) .
$$

Then, since $\left\{e_{1}, e_{2}, e_{5} \ldots, e_{4+s}\right\}$ is a local orthonormal basis of $\mathcal{X}(M)$, dual of $\left\{w^{1}, w^{2}, \eta_{1}, \ldots, \eta_{s}\right\}$, equation (5.9) follows from (5.14), (5.15) and (5.19).

Theorem 5.2 Let $M$ be a proper slant submanifold of an $S$-manifold

$$
\left(\widetilde{M}, f, \xi_{1}, \ldots, \xi_{s}, \eta_{1}, \ldots, \eta_{s}, g\right)
$$

with $\operatorname{dim} M=2+s, \operatorname{dim} \widetilde{M}=4+s$ and slant angle $\theta$. Suppose that there exists on $\widetilde{M}$ an $f$-structure $\bar{f}$ such that

$$
\left(\widetilde{M}, \bar{f}, \xi_{1}, \ldots, \xi_{s}, \eta_{1}, \ldots, \eta_{s}, g\right)
$$

is a metric $f$-manifold satisfying

$$
g\left(\left(\widetilde{\nabla}_{X} \bar{f}\right) Y, Z\right)=0
$$

for any $X, Y, Z$ normal to the structure vector fields. If $M$ is an antiinvariant submanifold with respect to this structure, then $M$ is a minimal submanifold of $\widetilde{M}$.

Proof. Let $\left\{e_{1}, \ldots, e_{4+s}\right\}$ be an adapted slant basis in the $S$-manifold

$$
\left(\widetilde{M}, f, \xi_{1}, \cdots, \xi_{s}, \eta_{1}, \cdots, \eta_{s}, g\right),
$$

being $\left\{e_{3}, e_{4}\right\}$ a local orthonormal frame of $T^{\perp} M$. Hence, since $M$ is an anti-invariant submanifold in

$$
\left(\widetilde{M}, \bar{f}, \xi_{1}, \ldots, \xi_{s}, \eta_{1}, \ldots, \eta_{s}, g\right)
$$

we have that $\left\{\bar{f} e_{1}, \bar{f} e_{2}\right\}$ is another local orthonormal basis of $T^{\perp} M$, by virtue of (3.1). Consequently, there exists a function $\varphi$ in $M$ such that:

$$
\begin{aligned}
& e_{3}=(\cos \varphi) \bar{f} e_{1}+(\sin \varphi) \bar{f} e_{2} \\
& e_{4}=-(\sin \varphi) \bar{f} e_{1}+(\cos \varphi) \bar{f} e_{2} .
\end{aligned}
$$

Consider $X \in \mathcal{L}$. Then, we get:

$$
\begin{aligned}
w_{3}^{4}(X)=g\left(\widetilde{\nabla}_{X} e_{3}, e_{4}\right)=X & (\cos \varphi) g\left(\bar{f} e_{1}, e_{4}\right)+X(\sin \varphi) g\left(\bar{f} e_{2}, e_{4}\right)+ \\
& +(\cos \varphi) g\left(\widetilde{\nabla}_{X} \bar{f} e_{1}, e_{4}\right)+(\sin \varphi) g\left(\widetilde{\nabla}_{X} \bar{f} e_{2}, e_{4}\right) .
\end{aligned}
$$


Now, since $w_{1}^{1}(X)=0, \bar{f} \xi_{\alpha}=0$ for any $\alpha=1, \ldots, s$ and $g\left(\bar{f} e_{4}, e_{4}\right)=0$, by using (5.20) and (5.21), we obtain:

$$
w_{3}^{4}(X)-w_{1}^{2}(X)=X \varphi=\mathrm{d} \varphi(X) .
$$

Now, consider any

$$
X=\widetilde{X}+\sum_{\alpha=1}^{s} \eta_{\alpha}(X) \xi_{\alpha} \in \mathcal{X}(M),
$$

with $\tilde{X} \in \mathcal{L}$. We find, by using (5.19) and (5.22) that:

$$
\begin{aligned}
w_{3}^{4}(X)-w_{1}^{2}(X) & =w_{3}^{4}(\tilde{X})-w_{1}^{2}(\tilde{X})+\sum_{\alpha=1}^{s} \eta_{\alpha}(X)\left(w_{3}^{4}\left(\xi_{\alpha}\right)-w_{1}^{2}\left(\xi_{\alpha}\right)\right)= \\
& =\mathrm{d} \varphi(\tilde{X})+2 \cos \theta \sum_{\alpha=1}^{s} \eta_{\alpha}(X) .
\end{aligned}
$$

But,

$$
\mathrm{d} \varphi(\widetilde{X})=\mathrm{d} \varphi\left(X-\sum_{\alpha=1}^{s} \eta_{\alpha}(X) \xi_{\alpha}\right)=\mathrm{d} \varphi(X)-\sum_{\alpha=1}^{s} \xi_{\alpha}(\varphi) \eta_{\alpha}(X)
$$

and, so:

$$
w_{3}^{4}-w_{1}^{2}=\mathrm{d} \varphi+\sum_{\alpha=1}^{s}\left(2 \cos \theta-\xi_{\alpha}(\varphi)\right) \eta_{\alpha} .
$$

Next, taking into account (5.9) we have:

$$
-\cot \theta\left\{\left(\operatorname{trace} \sigma^{3}\right) w^{1}+\left(\operatorname{trace} \sigma^{4}\right) w^{2}\right\}=\mathrm{d} \varphi-\sum_{\alpha=1}^{s} \xi_{\alpha}(\varphi) \eta_{\alpha} .
$$

On the other hand,

$$
\sigma_{11}^{3}=g\left(\sigma\left(e_{1}, e_{1}\right), e_{3}\right)=g\left(A_{e_{3}} e_{1}, e_{1}\right)=-g\left(\widetilde{\nabla}_{e_{1}} e_{3}, e_{1}\right)
$$

and from (5.20), (5.21) and since $\bar{f} e_{1}, \bar{f} e_{2} \in T^{\perp} M$, we get:

$$
\sigma_{11}^{3}=\cos \varphi g\left(\sigma\left(e_{1}, e_{1}\right), \bar{f} e_{1}\right)+\sin \varphi g\left(\sigma\left(e_{1}, e_{2}\right), \bar{f} e_{1}\right) .
$$

However, from (5.21) again:

$$
\bar{f} e_{1}=\cos \varphi e_{3}-\sin \varphi e_{4} .
$$

Consequently:

$$
\begin{aligned}
\sigma_{11}^{3} & =\cos ^{2} \varphi \sigma_{11}^{3}-\cos \varphi \sin \varphi \sigma_{11}^{4}+\cos \varphi \sin \varphi \sigma_{12}^{3}-\sin ^{2} \varphi \sigma_{12}^{4}= \\
& =\cos ^{2} \varphi \sigma_{11}^{3}-\sin ^{2} \varphi \sigma_{22}^{3},
\end{aligned}
$$

where we have used Lemma 5.1. 
Thus, since $\sigma_{\alpha \alpha}^{3}=0$, for any $\alpha=1, \ldots, s$ :

$$
\sin ^{2} \varphi\left(\operatorname{trace} \sigma^{3}\right)=0 \text {. }
$$

Analogously:

$$
\sin ^{2} \varphi\left(\operatorname{trace} \sigma^{4}\right)=0 .
$$

Now, let consider the following open subset of $M$ :

$$
U=\{x \in M / H(x) \neq 0\} .
$$

To conclude the proof, we only need to show that $U \neq \emptyset$. If it is not the case, then, in $U$,

$$
0 \neq H=\frac{1}{2+s} \operatorname{trace} \sigma=\frac{1}{2+s}\left(\left(\operatorname{trace} \sigma^{3}\right) e_{3}+\left(\operatorname{trace} \sigma^{4}\right) e_{4}\right),
$$

and so:

$$
\text { trace } \sigma^{3} \neq 0 \text { or trace } \sigma^{4} \neq 0 \text {. }
$$

This implies, by virtue of $(5.24)$ and $(5.25)$, that $\varphi \equiv 0(\bmod \pi)$ in $U$. But $\varphi$ is a continuous function, thus $\varphi \equiv 0$ in $U$. Hence, $\mathrm{d} \varphi=0$ and $\xi_{\alpha}(\varphi)=0$ in $U$, for any $\alpha=1, \ldots, s$. Then, from (5.23),

$$
\cot \theta\left(\left(\operatorname{trace} \sigma^{3}\right) w^{1}+\left(\operatorname{trace} \sigma^{4}\right) w^{2}\right)=0,
$$

and from (5.26), $\cot \theta=0$, which is a contradiction with the fact of $M$ being a proper slant submanifold. So, $U=\emptyset$ and $M$ is minimal.

Note that the above theorem holds, in particular, if

$$
\left(\widetilde{M}, \bar{f}, \xi_{1}, \ldots, \xi_{s}, \eta_{1}, \ldots, \eta_{s}, g\right)
$$

is an $S$-structure on $\widetilde{M}$ because, in such a case, for any $X, Y, Z \in \mathcal{X}(\widetilde{M})$, from (3.3) we find

$$
g\left(\left(\widetilde{\nabla}_{X} \bar{f}\right) Y, Z\right)=\sum_{\alpha=1}^{s}\left(g(f X, f Y) \eta_{\alpha}(Z)+\eta_{\alpha}(Y) g\left(f^{2} X, Z\right)\right),
$$

vanishing this expression if $Y, Z$ are normal to the structure vector fields. In fact, this would be the corresponding theorem to Theorem 4.2 of [4] which was proved by B.-Y. Chen in the Kaehlerian case. However, we have the following proposition: 
Proposition 5.1 Let $\left(\widetilde{M}, f, \xi_{1}, \ldots, \xi_{s}, \eta_{1}, \ldots, \eta_{s}, g\right)$ be an $S$-manifold. If there exists another $f$-structure $\bar{f}$ on $\widetilde{M}$ such that

$$
\left(\widetilde{M}, \bar{f}, \xi_{1}, \ldots, \xi_{s}, \eta_{1}, \ldots, \eta_{s}, g\right)
$$

is a metric $f$-manifold with $F_{\bar{f}}=\mathrm{d} \eta_{a}$, for any $\alpha=1, \ldots, s$, then $f=\bar{f}$.

Proof. The two fundamental 2-forms satisfy

$$
F_{f}=\mathrm{d} \eta_{a}=F_{\bar{f}}, \quad \text { for any } \alpha=1, \ldots, s .
$$

Then, for any $X, Y \in \mathcal{X}(\widetilde{M})$,

$$
g(X, f Y)=F_{f}(X, Y)=F_{\bar{f}}(X, Y)=g(X, \bar{f} Y)
$$

which implies $f Y=\bar{f} Y$, for any $Y \in \mathcal{X}(\widetilde{M})$.

Consequently, Theorem 5.2 is the best possible version of Chen's Theorem for $S$-manifolds, because there are not different compatible $S$-structures on the same manifold.

Finally, let us consider an example. Let

$$
\left(\mathbb{R}^{4+s}, f, \xi_{1}, \ldots, \xi_{s}, \eta_{1}, \ldots, \eta_{s}, g\right)
$$

be the usual $S$-structure on $\mathbb{R}^{4+s}$ (see [7] for more details) given by the following elements

$$
\begin{aligned}
& \eta_{\alpha}=\frac{1}{2}\left(\mathrm{~d} z^{\alpha}-\sum_{i=1}^{2} y^{i} \mathrm{~d} x^{i}\right), \xi_{\alpha}=2 \frac{\partial}{\partial z^{\alpha}} \\
& g=\sum_{\alpha=1}^{s} \eta_{\alpha} \otimes \eta_{\alpha}+\frac{1}{4}\left(\sum_{i=1}^{2}\left(\mathrm{~d} x^{i} \otimes \mathrm{d} x^{i}+\mathrm{d} y^{i} \otimes \mathrm{d} y^{i}\right)\right) \\
& f\left(\sum_{i=1}^{2}\left(X_{i} \frac{\partial}{\partial x^{i}}+Y_{i} \frac{\partial}{\partial y^{i}}\right)+\sum_{\alpha=1}^{s} Z_{\alpha} \frac{\partial}{\partial z^{\alpha}}\right)= \\
& =\sum_{i=1}^{2}\left(Y_{i} \frac{\partial}{\partial x^{i}}-X_{i} \frac{\partial}{\partial y^{i}}\right)+\sum_{\alpha=1}^{s} \sum_{i=1}^{2} Y_{i} y^{i} \frac{\partial}{\partial z^{\alpha}}
\end{aligned}
$$

where $\left(x^{1}, x^{2}, y^{1}, y^{2}, z^{1}, \ldots, z^{s}\right)$ are denoting the cartesian coordinates on $\mathbb{R}^{4+s}$. Define on $\mathbb{R}^{4+s}$ the $(1,1)$-tensor field $\bar{f}$ by:

$$
\begin{aligned}
& \bar{f}\left(\sum_{i=1}^{2}\left(X_{i} \frac{\partial}{\partial x^{i}}+Y_{i} \frac{\partial}{\partial y^{i}}\right)+\sum_{\alpha=1}^{s} Z_{\alpha} \frac{\partial}{\partial z^{\alpha}}\right)= \\
& =-X_{2} \frac{\partial}{\partial x^{1}}+X_{1} \frac{\partial}{\partial x^{2}}+Y_{2} \frac{\partial}{\partial y^{1}}-Y_{1} \frac{\partial}{\partial y^{2}}+\left(y^{2} X_{1}-y^{1} X_{2}\right) \sum_{\alpha=1}^{s} \frac{\partial}{\partial z^{\alpha}}
\end{aligned}
$$


It is easy to prove that

$$
\left(\mathbb{R}^{4+s}, \bar{f}, \xi_{1}, \ldots, \xi_{s}, \eta_{1}, \ldots, \eta_{s}, g\right)
$$

is a metric $f$-manifold. Moreover,

$$
\left(\widetilde{\nabla}_{X} \bar{f}\right) Y=\sum_{\alpha=1}^{s}\left(2 \eta_{\alpha}(X) \bar{f} f Y+\eta_{\alpha}(Y) \bar{f} f X+g(X, \bar{f} f Y) \xi_{\alpha}\right.
$$

for any $X, Y \in \mathcal{X}(\widetilde{M})$. Then, we have (5.20).

Now, consider the $(2+s)$-dimensional submanifold $M$ of $\mathbb{R}^{4+s}$ defined by

$$
x\left(u, v, t_{1}, \ldots, t_{s}\right)=2\left(u \cos \theta, u \sin \theta, v, 0, t_{1}, \ldots, t_{s}\right),
$$

for any $\theta \in(0, \pi / 2)$. Then, $M$ is a minimal proper slant submanifold in

$$
\left(\mathbb{R}^{4+s}, f, \xi_{1}, \ldots, \xi_{s}, \eta_{1}, \ldots, \eta_{s}, g\right)
$$

(see [3]) and an anti-invariant submanifold in

$$
\left(\mathbb{R}^{4+s}, \bar{f}, \xi_{1}, \ldots, \xi_{s}, \eta_{1}, \ldots, \eta_{s}, g\right) .
$$

\section{References}

[1] Blair, D.E.: Geometry of manifolds with structural group $\mathcal{U}(n) \times \mathcal{O}(s)$. J. Differential Geometry 4 (1970), 155-167.

[2] Cabrerizo, J.L., Carriazo, A., Fernández, L.M. AND Fernández, M.: Slant submanifolds in Sasakian manifolds. Glasg. Math. J. 42 (2000), 125-138.

[3] Carriazo, A.: New developments in slant submanifolds theory. In Applicable Mathematics in the Golden Age (Edited by J.C. Misra), 339-356. Narosa Publishing House, New Delhi, 2002.

[4] Chen, B.-Y.: Geometry of slant submanifolds. Katholieke Universiteit Leuven, Louvain, 1990.

[5] Chen, B.-Y.: Geometry of submanifolds. Pure and Applied Mathematics 22. Marcel Dekker, Inc., New York, 1973.

[6] Chen, B.-Y.: Slant immersions. Bull. Austral. Math. Soc. 41 (1990), no. 1, 135-147.

[7] Hasegawa, I., Okuyama, Y. And Abe, T.: On p-th Sasakian manifolds. J. Hokkaido Univ. Ed. Sect. II A 37 (1986), no. 1, 1-16. 
[8] Lotta, A.: Slant submanifolds in contact geometry. Bull. Math. Soc. Sci. Math. R. S. Roumanie (N.S.) 39 (1996), 183-198.

[9] YANO, K.: On a structure defined by a tensor field $f$ of type $(1,1)$ satisfying $f^{3}+f=0$. Tensor (N.S.) 14 (1963), 99-109.

Recibido: 9 de septiembre de 2002

Revisado: 24 de junio de 2003

Alfonso Carriazo

Departamento de Geometría y Topología

Facultad de Matemáticas

Universidad de Sevilla

Apartado de Correos 1160

41080-Sevilla, Spain

carriazo@us.es

Luis M. Fernández

Departamento de Geometría y Topología

Facultad de Matemáticas

Universidad de Sevilla

Apartado de Correos 1160

41080-Sevilla, Spain.

lmfer@us.es

María Belén Hans-Uber

Departamento de Geometría y Topología

Facultad de Matemáticas

Universidad de Sevilla

Apartado de Correos 1160

41080-Sevilla, Spain.

The authors are partially supported by the PAI project (Junta de Andalucía, Spain, 2002). 